\title{
Experimental demonstration of an All-Optical Interferometric Drop, Add, and Extract Multiplexer for OFDM Super-channel
}

\author{
Simon J. Fabbri ${ }^{(1,2, *)}$, Stylianos Sygletos ${ }^{(1)}$, Andreas Perentos ${ }^{(1)}$, Erwan Pincemin ${ }^{(3)}$, Kate Sugden ${ }^{(1)}$, and Andrew D. Ellis ${ }^{(1)}$ \\ ${ }^{1}$ Aston Institute of Photonic Technologies, Aston University, Birmingham, UK \\ ${ }^{2}$ Department of Physics, University College Cork, Cork, Ireland \\ ${ }^{3}$ France Telecom, Orange Labs Networks, 22307 Lannion, France \\ s.fabbri@aston.ac.uk
}

\begin{abstract}
:
The experimental implementation of an all-optical node able of routing a channel contained in an all-optical OFDM super-channel is presented. The extract function is performed through channel selection, reshaping and interferometric suppression.
\end{abstract}

\section{Introduction:}

The development of large high efficiency super-channels is driven by the continuous growth of optical network capacity demand. When point to point links are approaching the fundamental limit of standard fibre, the efficient use of the entire transmission window for optical network will only be achieved through flexible superchannels compatible with Reconfigurable Optical Add Drop Multiplexers (ROADMs).

For Nyquist WDM and DSP generated OFDM signals, ROADMs based on high quality optical filters have been investigated for sub-channel routing [1], [2]. When multiple ROADMs were cascaded, unless wide guard bands are applied, penalties were observed due to the tight filtering, presenting a trade-off between spectral efficiency and ROADM cascadability. This approach of course is incompatible with super-channels with overlapping spectra, such as OFDM, Nyquist WDM, Time Frequency Packing, and All-Optical OFDM (AO-OFDM). To avoid the limitation inherent to optical filtering as a means to extract components from a super-channel, interferometric suppression has been proposed [3], [4] where a carbon copy of the channel to be extracted is obtained and then combined out of phase with the superchannel. The proposed schemes are based on multiple medium interferometer, where the superchannel is maintained in the optical domain while the channel to be extracted has to be received by a complete electronic system. After the analogue to digital conversion and digital signal processing, the channel to be remove from the superchannel needs to be reemitted in the optical domain with the appropriate phase to obtain the interferometric suppression. Proof of concept of this DSP scheme has been implemented emulating a two medium interferometer [5], using a coherent receiver and high-speed transmitter but the difficulties of closing the multiple medium interferometer were not addressed

Recently, we have designed [6] and implemented [7], [8] a new all-optical architecture, named Terabit Interferometric Drop, Add, and Extract (TIDE) multiplexer, to provide switching of subchannels within an AOOFDM signal band. In this paper we discuss its performance with a QPSK super-channel.

\section{Terabit Interferometric Add, Drop, and Extract test setup:}

In the proposed scheme, the switching is performed by coherent subtraction of the signal to be extracted and the addition of the new through an all fibre interferometer. This approach requires the demultiplexing of the channel to be extracted from the super-channel followed by crosstalk-free reconstruction. First, the channel is demultiplexed from the super-channel using matched optical filter based on an optical FFT based demultiplexer. Whilst an ideal system has zero crosstalk at the decision point, crosstalk away from the optimum sampling instant, plus additional crosstalk due to imperfect filtering and finite bandwidth transmitter needs to be rejected. To facilitate this crosstalk rejection an optical sampler based on an optical gate is used to select approximately $10 \%$ of the dropped channel bit for each symbol. The optimum sampling window is a compromise between noise sampling and crosstalk leakage. The third function is the reshaping of the sampled signal to match the channel to be dropped embedded in the super-channel which propagates in the second arm of the fibre interferometer. Then, with accurate channel reshaping and appropriate phase control, when the reshaped signal is interfered with the super-channel the channel is suppressed without affecting the adjacent channels. A new channel may be added in the spectral space created within the superchannel.

Fig. 1 shows the simplified view of the experimental implementation of the TIDE node. The top part of the figure is composed of three parts representing the All-Optical OFDM transmitter in the green box, the extract function in the blue box, and the add function in the orange box. The transmitter delivered ten $20 \mathrm{Gbit} / \mathrm{s}$ QPSK channels, with a channel spacing of $10 \mathrm{GHz}$. The extract function was built around a fibre Mach-Zehnder interferometer (MZI). Path A of the interferometer was a through path for the superchannel with control of the optical power, phase and delay. Path B contained the first optical FFT filter, implemented by two cascaded asymmetric MZIs and a $50 \mathrm{GHz}$ interleaver; an optical sampling gate with a 12 ps gating window with $35 \mathrm{~dB}$ extinction ratio based on two cascaded MZM; and the final iFFT filter implemented using two further asymmetric MZIs followed by a sharp commercially available optical filter. A more complete description can be 


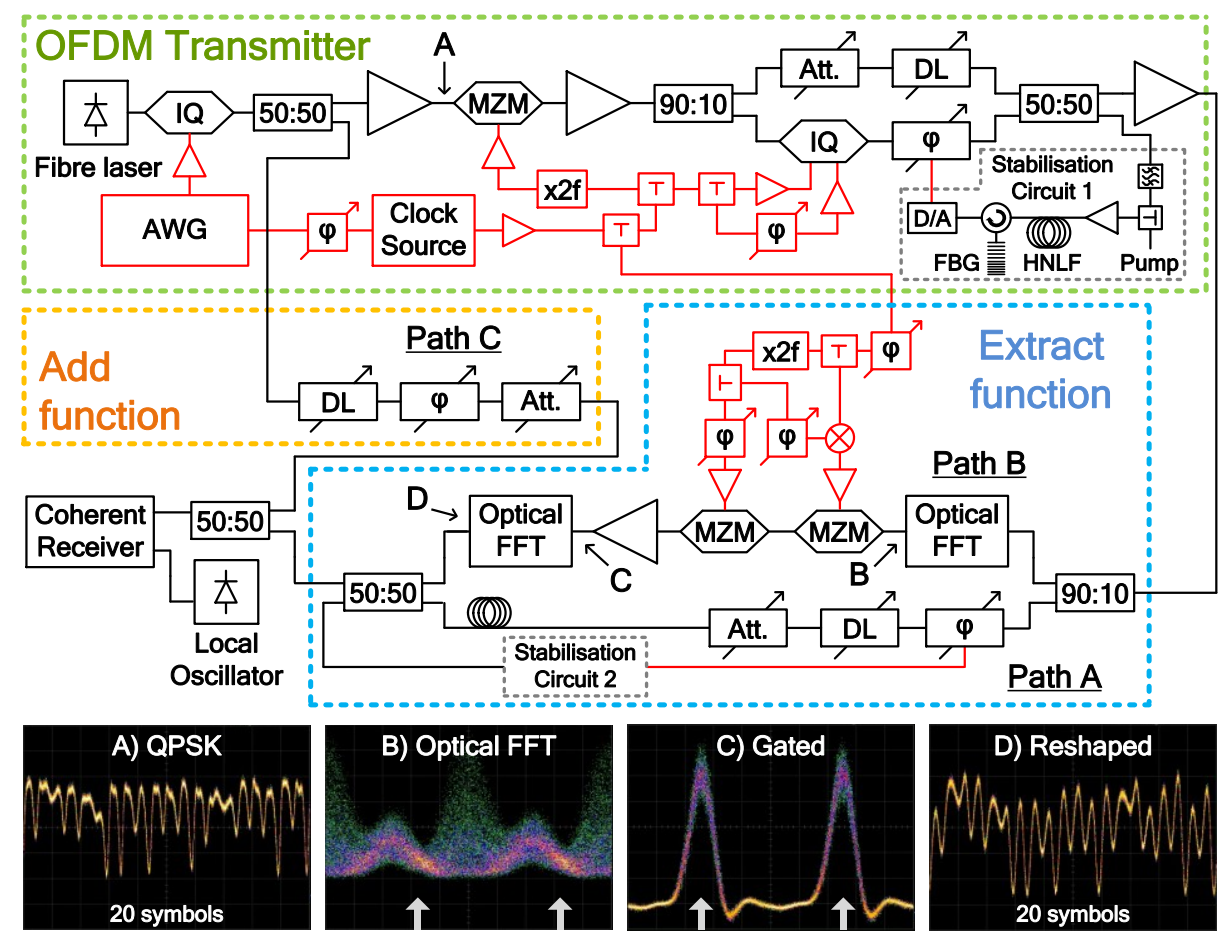

Fig1: Top: Experimental setup of the AO-OFDM transmitter (top, green frame) and of the proposed all optical ROADM with drop and extract function (bottom, blue frame, path $\mathrm{A} \& \mathrm{~B}$ ) and emulation of the add function (middle, orange frame, path C). Polarisation controllers and monitoring couplers not pictured for clarity. Black and red lines signify optical and electrical paths respectively. AWG: Arbitrary function generator, MZM: Mach-Zehnder modulator, DL: tunable delay line, $\varphi$ : tunable phase shifter, Att.: tunable attenuator, IQ: dual parallel MZM, HNLF: highly nonlinear fibre, FBG: fibre Bragg grating, D/A: digital/analog converter, triangles represent optical or electrical amplifiers.

Bottom: Intensity eye diagrams at various points: A) Time trace of individual channel before superchannel multiplexing, B) Intensity eye diagrams of demultiplexed channel after optical channel selection through optical FFT filter, C) Intensity eye diagrams of sampled channel after optical gating, and D) Time trace of reshaped channel prior to interferometric extract operation. Arrows represent the center of the eye. Eyediagrams window equals to two bit period. Oscilloscope electrical bandwidth: $30 \mathrm{GHz}$.

found in [8]. When the two paths A) and B) are recombined out of phase, the channel to be dropped was suppressed by more than $10 \mathrm{~dB}$ from the superchannel (observation limited by the $2 \mathrm{GHz}$ resolution of the spectrum analyser). The inserts in Fig.1 shows the modification of the signal along the reshaping path. Insert A) illustrated the time trace of the QPSK source signal used for the superchannel. B) shows the temporal distribution of crosstalk leaking after channel selection whereas the C) presents a clear RZ-QPSK signal freed from crosstalk after optical sampling. Finally, D) shows the reshaped channel before the interferometric suppression, and should be compared to signal A). When a new QPSK channel was added, performance levels measured at the coherent receiver were all observed to be above the 7\%-overhead Hard Decision FEC limit. The impact on the adjacent channels was limited to a few $\mathrm{dB}$ despite the overlapping spectra nature of AO-OFDM. Improved filters along with full stabilisation and/or integration would open the way for higher order modulation.

\section{Conclusion:}

We experimentally implemented a new all-optical node for AO-OFDM based on an interferometric structure. The standard node functions, channel drop, extraction, and insertion were achieved whilst the signal was kept in the optical domain. With the implementation of the TIDE node presented here, routing superchannel carrying dual-quadrature formats, the way for guardband-free all-optical flexible transport is proven possible.

\section{Acknowledgments:}

The work has been supported by the EU-ICT project FOX-C, grant number " 318415 " and by the Marie Currie-IEF project ARTISTE, grant number "330697".

\section{References:}

[1] E. Pincemin et al., J. Lightw. Technol., vol. 32, no. 12, 2014

[2] A. Ghazisaeidi et al., in Proc. ECOC 2013, pp. OTu3B-6

[3] M. G. Taylor, U.S. Patent 8,050,564, issued Nov.1, 2011

[4] P. J. Winzer, J. Lightw. Technol., vol. 31, no. 11, 2013

[5] T. Richter et al., in Proc. OFC/NFOEC 2014, Th5B-6

[6] S. Sygletos et al., Opt. Express, vol. 23, no. 5, 2015

[7] S. J. Fabbri et al., in Proc. ECOC 2014, We.1.5.2

[8] S. J. Fabbri et al., J. Lightw. Technol., vol. 33, no. 7, 2015 